\title{
BIOMIMETIC SUPERHYDROPHOBIC SURFACES USING VIRAL NANOTEMPLATES FOR SELF-CLEANING AND DROPWISE CONDENSATION
}

\author{
M. McCarthy ${ }^{1 *}$, R. Enright ${ }^{1,2}$, K. Gerasopoulos ${ }^{3}$, J.N. Culver ${ }^{3}$, R. Ghodssi ${ }^{3}$, and E.N. Wang ${ }^{1}$ \\ ${ }^{1}$ Massachusetts Institute of Technology, Cambridge, Massachusetts, USA \\ ${ }^{2}$ University of Limerick, Limerick, Ireland, ${ }^{3}$ University of Maryland, College Park, Maryland, USA
}

\begin{abstract}
We report the fabrication and characterization of biomimetic superhydrophobic surfaces synthesized using self-assembly and metallization of the Tobacco mosaic virus (TMV) onto micropillar arrays. A novel biofabrication process was used to accurately recreate the hierarchical surface structures of the lotus leaf (Nelumbo nucifera) and has provided key insights into the role of both nanoscale and microscale features on water repellency of aquatic and wetland plant leaves under droplet impact. The biomimetic surfaces have been demonstrated to be robust, where droplets rebound with impact velocities greater than $4.3 \mathrm{~m} / \mathrm{s}$. Additionally, there is a two-fold increase in the steady state condensation heat transfer coefficient across the biomimetic surfaces as compared to planar ones.
\end{abstract}

\section{INTRODUCTION}

Superhydrophobic surfaces, with static contact angles greater than $150^{\circ}$ and droplet hystereses less than $10^{\circ}$, resist wetting and exhibit self-cleaning properties. Such properties are ideal for coatings on buildings, solar cells, and textiles, as well as drag reduction and increased heat transfer via drop-wise condensation $[1,2]$. In nature, a wide array of wetland and aquatic plant leaves exhibit self-cleaning properties and resist wetting upon the impact of rainfall. Due to the abundance of water, these wetland plants do not rely on the intake of moisture through their leaves to hydrate. In fact, their superhydrophobic properties are a necessity for survival. Shedding water from the surface dramatically increases the uptake of $\mathrm{CO}_{2}$ for photosynthesis, and these self-cleaning abilities reduce the formation of bacteria and fungi that would otherwise thrive in such hot moist climates [3,4]. Significant efforts have focused on mimicking the naturally occurring structures of the lotus leaf, which demonstrates superhydrophobic self-cleaning properties [4]. However, existing fabrication methods have limited the ability to accurately mimic both the surface structures and resulting water-repellent behavior of the lotus under droplet impact.

In this work, a novel method for creating biomimetic hierarchical surfaces based on the Tobacco mosaic virus (TMV) is implemented to study the role of length scales on the anti-wetting behavior of superhydrophobic surfaces. The TMV is a plant virus, measuring $300 \mathrm{~nm}$ in length and $18 \mathrm{~nm}$ in diameter, which is benign to humans and can be genetically engineered to have enhanced binding properties. The use of the TMV for the synthesis of biomimetic water-repellent surfaces has several specific advantages over existing techniques. Specifically, the existence of functional groups enables self-assembly and directed patterning over a wide range of materials (metals, ceramics and polymers) and geometries [5].

\section{FABRICATION}

Biomimetic surfaces inspired by the lotus leaf have been fabricated using self-assembly and metallization of the Tobacco mosaic virus onto micropillar arrays. A photo-definable negative resist (SU-8 10, Microchem) was spin-coated to a thickness of
$15 \mu \mathrm{m}$ on a silicon wafer and exposed to create micropost arrays The wafer was then diced into individual $2 \mathrm{~cm} \times 2 \mathrm{~cm}$ die. The die were placed in a phosphate buffer solution $(\mathrm{pH} 7)$ containing the $\mathrm{TMV}$ at a concentration of $0.1 \mathrm{mg} / \mathrm{mL}$ and allowed to incubate overnight where the virus self-assembled on the exposed silicon and SU-8 surfaces. After TMV assembly, the surface-exposed cysteines of the virus were activated with a palladium catalyst in a solution prepared by mixing a palladium salt with phosphate buffer. The samples were then coated with nickel in an electroless plating solution in which they were immersed for 3-5 minutes. The catalyst solution was prepared by dissolving $29 \mathrm{mg}$ of $\mathrm{NaPdCl}_{4}$ in $10 \mathrm{~mL}$ of DI water. The nickel plating solution was prepared by mixing $0.6 \mathrm{~g} \mathrm{NiCl}_{2}, 0.45 \mathrm{~g}$ Glycine, $1.5 \mathrm{~g} \mathrm{Na}_{2} \mathrm{~B}_{4} \mathrm{O}_{7}, 0.77 \mathrm{~g} \mathrm{DMAB}$ and $25 \mathrm{~mL}$ of DI water and stirring until solution turns to $\mathrm{pH} 7$.
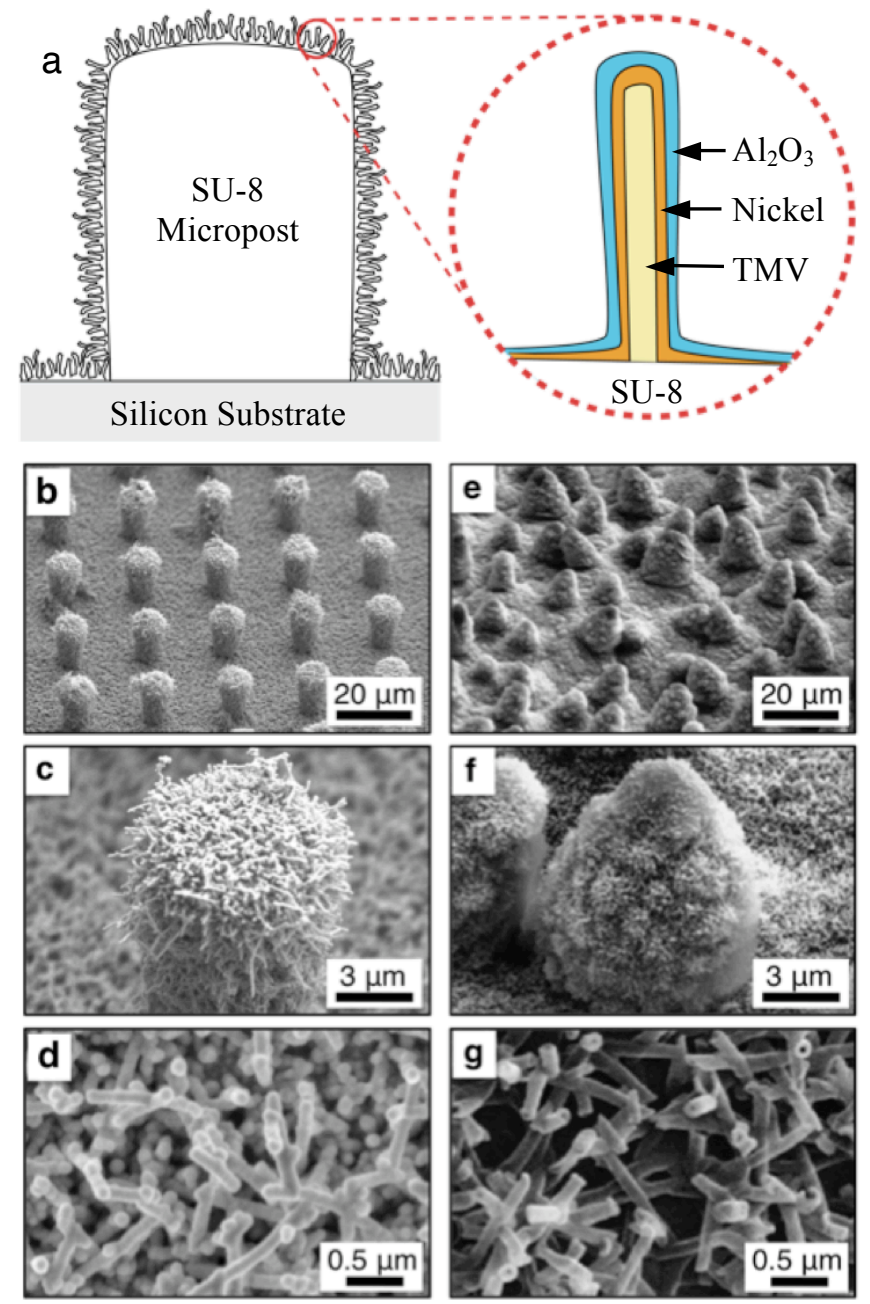

Figure 1: (a) Cross sectional schematic of the hierarchical surface structures and a direct comparison of $(b-d)$ the biomimetic surfaces with (e-g) the naturally occurring lotus leaf [3]. 
a Flat surface

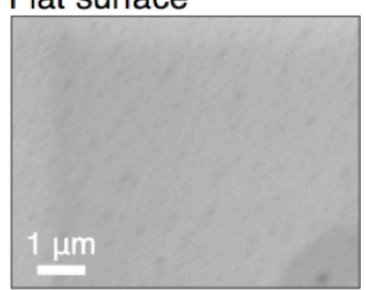

d Nanostructured surface

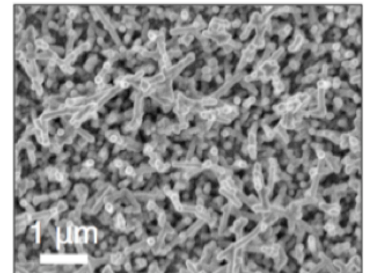

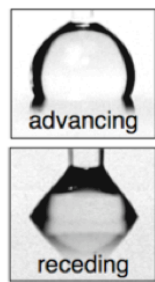

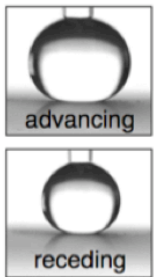

b Microstructured surface $(\mathrm{S}=0.13)$

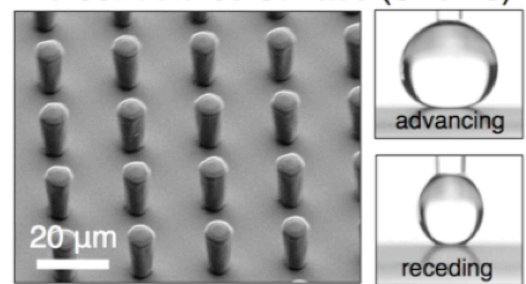

e Hierarchical surface $(S=0.13)$

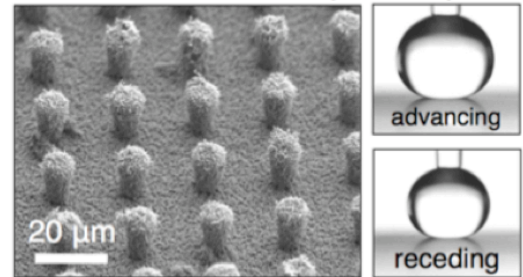

c Microstructured Surface $(\mathrm{S}=0.38)$

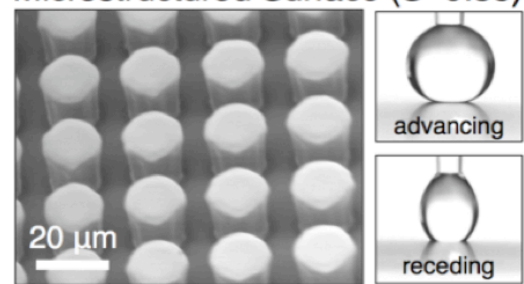

$f$ Hierarchical Surface $(S=0.38)$

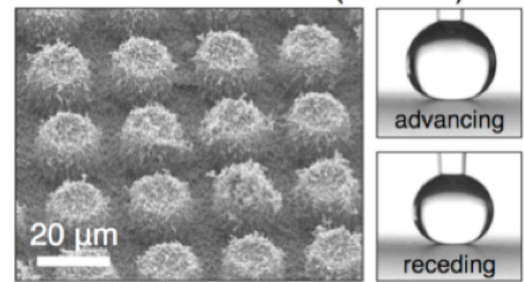

Figure 2: SEM images of experimentally characterized surfaces and optical images of their advancing and receding contact angles. (a) Flat surface, $(b, c)$ microstructured surfaces with $15 \mu \mathrm{m}$ tall posts spaced $20 \mu \mathrm{m}$ apart with diameters and solid fractions of (b) $d=8 \mu \mathrm{m}$, $S=0.13$ and (c) $d=14 \mu \mathrm{m}, S=0.38$, (d) nanostructured surface, and (e,f) hierarchical surfaces with 15 um tall posts (with nanostructures) spaced $20 \mu \mathrm{m}$ apart with diameters and solid fractions of $(e) d=8 \mu \mathrm{m}, S=0.13$ and $(f) d=14 \mu \mathrm{m}, S=0.38$.

After metallization, the surfaces were functionalized through atomic layer deposition (ALD) of $\mathrm{Al}_{2} \mathrm{O}_{3}$ followed by vapor-phase deposition of silane to achieve superhydrophobic properties. A uniform $15 \mathrm{~nm}$ thick $\mathrm{Al}_{2} \mathrm{O}_{3}$ was deposited, which also provides a conformal coating to the nanoscale features of the virus. A silane monolayer was formed onto the $\mathrm{Al}_{2} \mathrm{O}_{3}$ surface at room temperature with the samples exposed to vapor-phase (Tridecofluoro-1,1,2,2Tetrahydrooctyl)-1-Trichlorosilane for 40 minutes.

Figure 1(a) shows a cross-sectional schematic of the resulting micro- and nano-scale surface structures while Fig. 1(b-g) provides a side-by-side comparison of the biomimetic and naturally occurring surfaces at various length scales. As can be seen, selfassembly of the TMV provides consistent and conformal coverage of the polymer microstructures. This is particularly important for the current work, where not only the scale of the nanostructure is well mimicked, but also the shape and orientation, resulting in surfaces that closely resemble the lotus leaf. This has implications on both the physics of water-repellency as well as the viability of this nanomanufacturing technique for large-scale production.

Figure 2 shows the six surfaces fabricated for the current work. Flat surfaces both with (Fig. 2(d)) and without (Fig. 2(a)) viral nanostructures, as well as microstructured (Fig. 2(b,c)) and hierarchically structured (Fig. 2(e,f)) surfaces with two different solid fractions, have been synthesized and experimentally characterized. The solid fraction of the surfaces is calculated as $S=\pi d^{2} / 4 L^{2}$, where $d$ and $L$ are the pillar diameter and center-tocenter spacing, respectively.

\section{CHARACTERIZATION}

Droplet contact angle measurements and droplet impingement imaging were obtained using a high-speed camera (Phantom v7.1, Vision Research) and image-processing software (ImageJ). A micropump and controller (Micro4 Syringe Pump, World Precision Instruments) was used to dispense and control water droplets.

\section{Contact Angle Measurements}

Superhydrophobic surfaces are defined as those with static contact angles greater than $150^{\circ}$, contact angle hystereses of less than $10^{\circ}$, and roll-off tilt angles typically less than $2^{\circ}$. Contact angle hysteresis is defined as the difference between the advancing and receding contact angles, and the roll-off tilt angle is the angle of a tilted surface at which a droplet will roll off. These three values are inter-related and collectively used to determine a surfaces ability to demonstrate self-cleaning behavior.

Static contact angle measurements were obtained by applying single $10 \mu \mathrm{L}$ droplets to the sample surface and evaluating the apparent contact angle using image processing software. Advancing and receding contact angles were measured by increasing and decreasing the volume of a droplet on the sample surfaces while capturing images (Fig. 2). The nanostructured and hierarchical surfaces demonstrated roll-off angles below the accuracy of the measurement capabilities $\left(<0.25^{\circ}\right)$, and at no time during the testing of these surfaces could a droplet be maintained in static equilibrium. Accordingly, the static contact angle for nanostructured and hierarchical surfaces was taken to be the average of the advancing and receding contact angles. This is an acceptable approximation as the contact angle hysteresis is smaller than the accuracy of the measurement method. Results for static contact angle and contact angle hysteresis are shown in Fig. 3, for each fabricated sample. Both the nanostructured and hierarchical surface are superhydrophobic with static contact angles over $170^{\circ}$ and hystereses less than $2^{\circ}$.

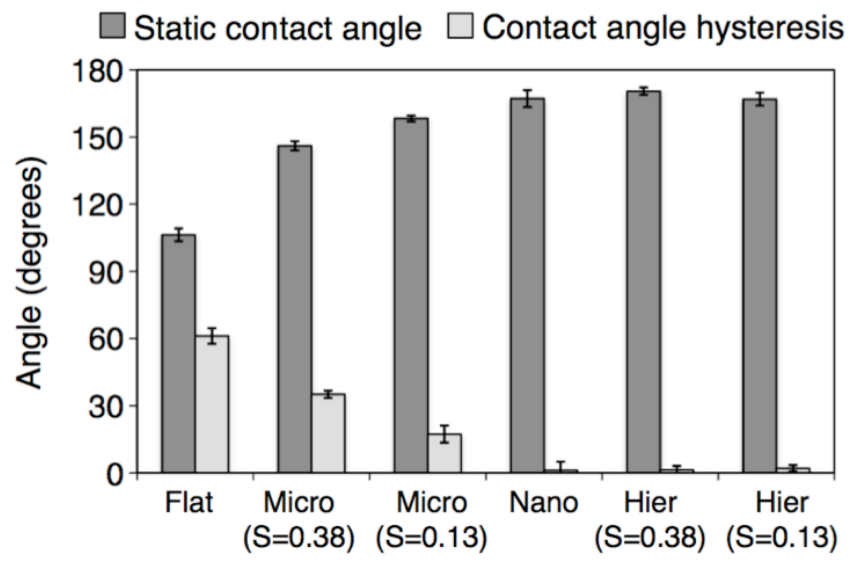

Figure 3: Experimental results of static contact angle and contact angle hysteresis for each fabricated sample shown in Fig. 2. 
These results raise interesting questions about the need for hierarchical structures in superhydrophobic water-resistant surfaces. If superhydrophobicity can be achieved with nanoscale features alone, why do self-cleaning aquatic and wetland plant leaves invariably have hierarchical surface structures [3]? The answer lies in the role of each length scale on water-repellency under droplet impact.

\section{Droplet Impact Wetting}

The relationship between contact angle and surface roughness is predicted by the theories of Cassie and Baxter, and Wenzel [5]. Cassie and Baxter's theory governs the behavior of static droplets resting on top of the surface roughness. Vapor pockets are present underneath the liquid resulting in a composite liquid-vapor-solid interface. In Wenzel's model, the droplet has completely penetrated the roughness and no vapor pockets are present. A droplet in the Wenzel state pins to the surface structures and resists droplet motion. While the Cassie state is statically stable, a droplet can be forced into the Wenzel state by overcoming an energy barrier that exists between the two states [5]. This transition from Cassie to Wenzel can occur during droplet impact and will be destructive to self-cleaning properties, making the dynamics of impingement crucial for robust self-cleaning surfaces. Accordingly, the wetting of superhydrophobic surfaces under droplet impact has been experimentally investigated.

To study the role of dual length scales on droplet impingement, $10 \mu \mathrm{L}$ droplets $(2.7 \mathrm{~mm}$ diameter $)$ were dropped from heights of $0.5-100 \mathrm{~cm}$ on each sample, resulting in impact velocities of $0.2-4.3 \mathrm{~m} / \mathrm{s}$. High speed imaging of droplet impacts on various surfaces are shown in Fig. 4. Figure 5(a) shows the critical impact velocity, $V_{C}$, at which wetting of a surfaces is first observed. At speeds higher than this value, the droplet (or some fraction of the droplet) remains attached, signifying a transition to the Wenzel state. Figure 5(b) shows the critical kinetic energy, $E_{C}$, of the droplet at which transition is first observed, defined as

$$
E_{C}=0.5 m V_{C}^{2}
$$

where $m$ is the mass of the droplet.
The microstructured surfaces wet at relatively low critical velocities while the nanostructured surface transitions to a wetted state at $2.7 \mathrm{~m} / \mathrm{s}$. Figure $4(\mathrm{a}, \mathrm{b})$ shows high-speed images of droplets impacting microstructured $(\mathrm{S}=0.38)$ and nanostructured surfaces above their critical velocities. Transition is seen for both; a large pinned droplet remains on the microstructured surface for a velocity of $2.1 \mathrm{~m} / \mathrm{s}$, and a small pinned droplet remains on the nanostructured surface for a velocity of $4.3 \mathrm{~m} / \mathrm{s}$.
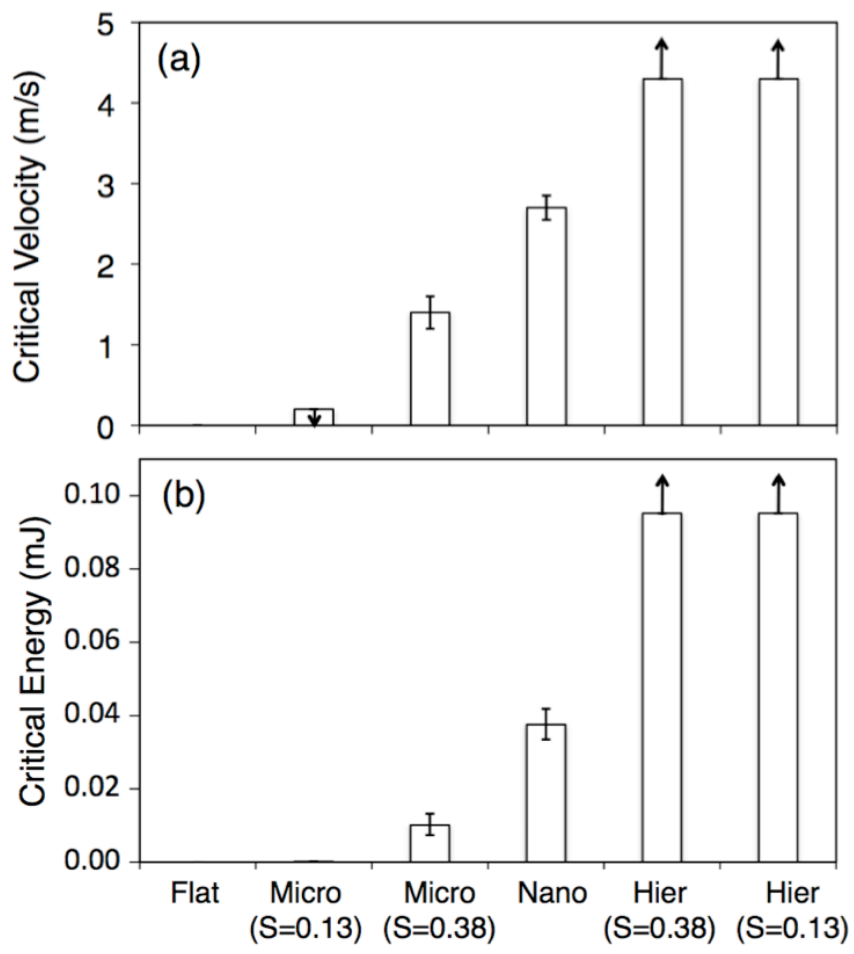

Figure 5: Droplet impact wetting results showing (a) the critical impact velocity, $V_{C}$, and (b) the critical impact energy, $E_{C}$, required to wet each fabricated surface structure.

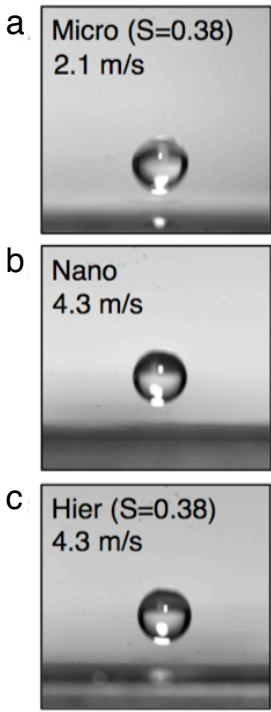

$0 \mathrm{~ms}$
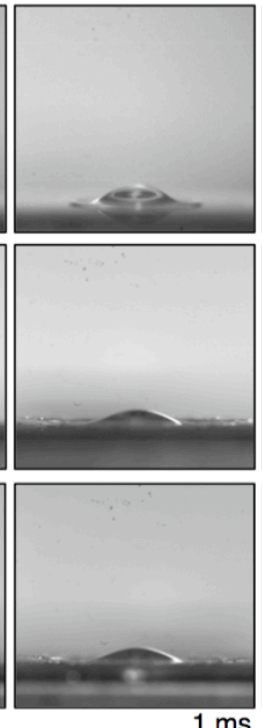

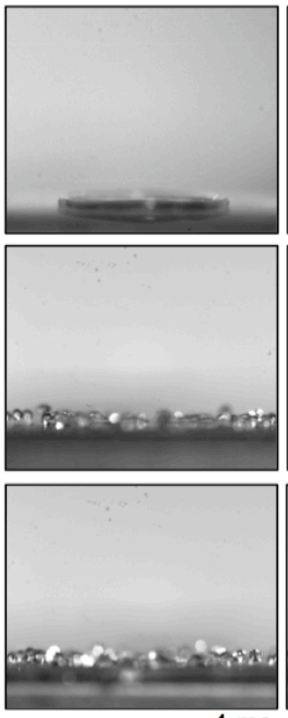

$4 \mathrm{~ms}$

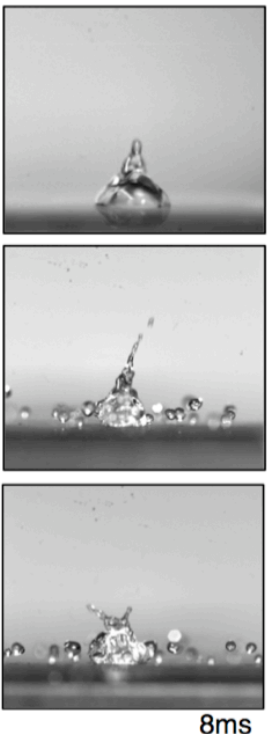

$8 \mathrm{~ms}$
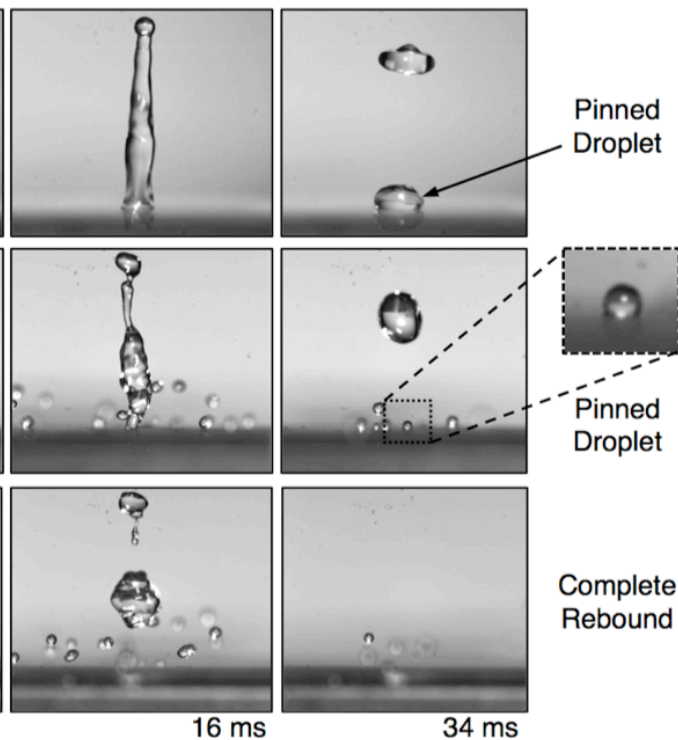

Figure 4: High-speed imaging of $10 \mu \mathrm{L}$ droplets impacting (a) the microstructured surface with a solid fraction of $S=0.38$ and an impingement velocity of $2.1 \mathrm{~m} / \mathrm{s}$, showing a large portion of the droplet wetted to the surface and partial rebound, (b) the nanostructured surface with a velocity of $4.3 \mathrm{~m} / \mathrm{s}$ showing partial wetting and break-up into satellite droplets, and (c) the hierarchical surface with a solid fractions of $S=0.38$, showing complete rebound and break-up into satellite droplets. 
The hierarchical surface shows complete rebound and breakup of the impinging droplets for all achievable speeds (Fig. 4(c)). While the critical velocities of the hierarchical surfaces have not been determined, it can be seen that the critical kinetic energy for the hierarchical transition is notably higher than the sum of its nano and microscale components (Fig. 5(b)). For the maximum speed considered $(4.3 \mathrm{~m} / \mathrm{s})$, the critical kinetic energy for hierarchical transition is shown to be at least twice as large as the sum of its components.

These counterintuitive results can be explained by considering the effects of compressibility on the impact pressure of impinging droplets. As a spherical droplet impacts a perfectly flat surface, a compressible no-flow region is generated in the droplet, resulting in large pressures associated with the compressed fluid [7]. This compressibility event occurs over a circular area on the order of tens of microns for millimeter-scale droplets falling at terminal velocities [7]. This critical length scale is identically matched by the microscale component of hierarchical structures found in aquatic and wetland plants and suggests that each length scale plays a distinct role in water repellency under droplet impact. It is proposed here that the microstructures have a destructive effect on the generation and propagation of the large pressures associated with compression, while the nanostructures provide a large antiwetting Laplace pressure resisting transition to a wetted state.

\section{Dropwise Condensation}

In addition to biomimetic self-cleaning behaviors, robust superhydrophobic surfaces can be implemented for the enhancement of condensation heat transfer rates. During the condensation process, water vapor changes phase into liquid on a sub-cooled surface. The liquid water forming on this surface results in an increased thermal resistance between it and the condensing vapor, which can greatly reduce the heat transfer coefficient of the process. Superhydrophobic surfaces are promising for the realization of drop-wise condensation heat transfer, where the condensing liquid does not wet the interface but instead forms into small droplets that roll off of the surface. This eliminates the existence of an insulating water film and has the potential to increase heat transfer coefficients by a factor of ten.

The condensation heat transfer performance of the hierarchical structures was evaluated by exposing the surface to a jet of water vapor while controlling the backside temperature using a thermoelectric module and active cooling. Figure 6 shows a sample being tested at a $45^{\circ}$ incline, while Fig. 7 shows preliminary experimental results for the hierarchical and flat surfaces. A two-fold increase in heat transfer coefficient was observed for the superhydrophobic surfaces as compared to flat samples with identical surface chemistries.

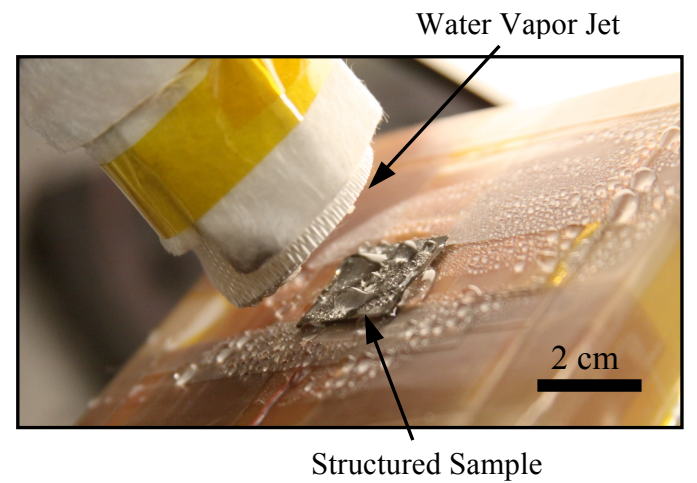

Figure 6: Image of the condensation process across a structured sample subject to a jet of condensing water vapor.

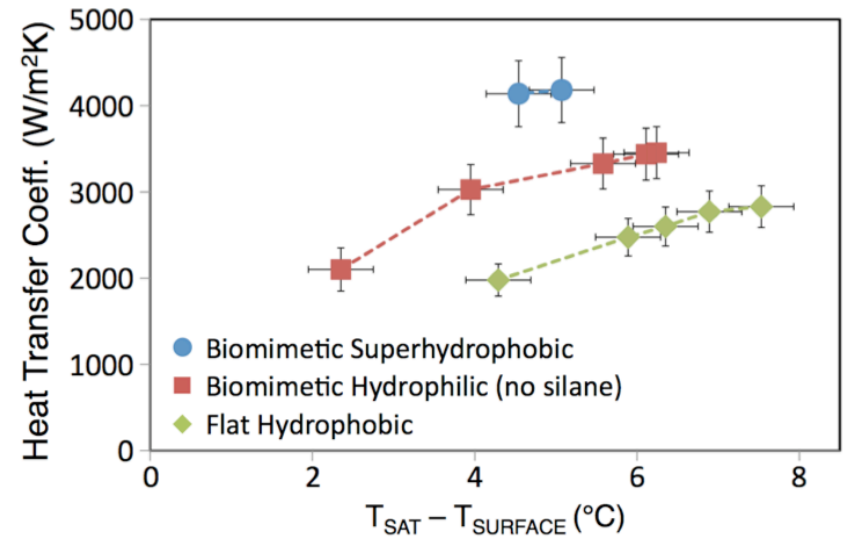

Figure 7: Experimental results of condensation heat transfer coefficient as a function of sub-cooling temperature.

\section{CONCLUSIONS}

Biomimetic surfaces inspired by the hierarchical structures of the lotus leaf have been fabricated using self-assembly and metallization of the Tobacco mosaic virus onto micropillar arrays. Wetting properties of the biomimetic superhydrophobic surfaces were evaluated through contact angle measurements, droplet impingement testing, and drop-wise condensation heat transfer studies. Static contact angles of over $170^{\circ}$ and contact angle hystereses of less than $2^{\circ}$ have been achieved. While surface hierarchy has little effect on the wetting of static droplets, it has been shown that hierarchical structures play an important role in water repellency under droplet impact. In addition, a two-fold increase in condensation heat transfer coefficient has been achieved with biomimetic surfaces as compared to flat surfaces.

By investigating the wetting mechanisms of biological structures, deeper insights into the design of high-performance thermal-fluidic systems can be gained. This work serves as a basis for developing robust superhydrophobic structures for selfcleaning surfaces, drop-wise condensation heat transfer, and drag reduction in both microscale and macro scale applications.

\section{REFERENCES}

[1] X. Feng, et al., "Design and Creation of Superwetting and Antiwetting Surfaces", Advanced Materials, 18, 3063 (2006).

[2] J. Boreyko, et al., Self-Propelled Dropwise Condensate on Superhydrophobic Surfaces", Phys. Rev. Letters, 103, 184501 (2009).

[3] K. Koch, et al., "Multifunctional Surface Structures of Plants: An Inspiration for Biomimetics", Prog. in Mat. Science 54, 137 (2009).

[4] A. Solga, et al., The Dream of Staying Clean: Lotus and Biomimetic Surfaces", Bioinspiration and Biomimetics, 2, S126 (2007).

[5] K. Gerasopoulos, et al., "Biofabrication Methods for the Patterned Assembly and Synthesis of Viral Nanotemplates", Nanotechnology, 21, (2010).

[6] A. Lafuma, et al., "Superhydrophobic States", Nature Mat., 2, (2003).

[7] O.G. Engel, "Damage Produced by High-Speed Liquid-Drop Impacts”, J. Appl. Phys. 44, 692 (1973).

\section{CONTACT}

*M. McCarthy, tel: +1-617-253-6401, mattmcc@mit.edu 